\title{
An X-Band Front-End Module Using HTS Technique for a Commercial Dual Mode Radar
}

\author{
Dow-Chih Niu, Tung-Wuu Huang, Hao-Jung Lee, and Chi-Yang Chang, Member, IEEE
}

\begin{abstract}
A front end module of receiver has been designed to eliminate the interference between the tracking and searching radar used in commercial radar application. The module is located at the receiving path of the searching radar. The proposed module includes two parts, the HTS superconducting filter and cryogenic low noise amplifier. The bandwidth of the eight-resonator HTS hairpin filter is $2 \%$ at the $\mathrm{X}$-band frequency. Low insertion loss (below1 dB) and good skirt rejection (down to $60 \mathrm{~dB}$ at $50 \mathrm{MHz}$ from the pass-band edge) are obtained. The two-stage low noise amplifier is designed by commercial low noise HEMT devices, NE321000. Two Lange couplers are located at output and input port of each amplifier stage to improve the return loss and to make LNA easily match to optimal low noise impendence. The cryogenic amplifier shows $30 \mathrm{~dB}$ gain and $0.4 \mathrm{~dB} \mathrm{NF}$ at $77 \mathrm{~K}$ in X-band.

A very high rejection is necessary for the elimination of unwanted spurious signals. The acceptable specification is $110 \mathrm{~dB}$ down at $500 \mathrm{MHz}$ away from the band-edge of the filter. In general, the skirt rejection of a hairpin HTS filter is pretty good near the pass-band edge, but may become worse far from the pass-band edge. To meet the requirement of this commercial radar application, a high rejection suspended stripline filter is put after the low noise amplifier. The whole module shows an maximal NF of $1.5 \mathrm{~dB}$, a gain of $25 \mathrm{~dB}$ around pass-band frequencies, and a rejection of better than $110 \mathrm{~dB}$ at $500 \mathrm{MHz}$ away from the band edge of the filter. It totally solves the interference problem in this dual mode radar.
\end{abstract}

Index Terms-Cross-coupled hairpin filter, cryogenic low noise amplifier, HTS filter, RF front end, searching and tracting radar.

\section{INTRODUCTION}

$\mathbf{I}$ NTERFERENCE between tracking and searching radar is the most serious problem for dual mode radar, especially, when the frequencies are close to each other. The best way to eliminate the interference is to put a high $\mathrm{Q}$ narrow band filter with high stop band rejection in the front of the receiver port. In general the narrow band filter provides high insertion loss. It also increases the system NF. Conventional solutions with increased Q-value are too bulky. As a result, tracking and searching radar frequencies must be spaced far apart in most dual mode radar.

High temperature superconductor filters have high Q characteristics. A filter could be designed to have very large skirt rejection and negligible insertion loss. It can eliminate the unwanted interference and would not increase the system noise figure.

Manuscript received October 3, 2004

D.-C. Niu, T.-W. Huang, and H.-J. Lee are with the Chung-Shan Institute of Science and Technology, Tao-Yuan, Taiwan, R.O.C. (e-mail: dcniu@ hotmail. com).

C.-Y. Chang is with the Department of Communication Engineering, National Chiao-Tung University, Hsin-Chu, Taiwan, R.O.C.

Digital Object Identifier 10.1109/TASC.2005.850184
The proposed front end module includes two parts, the HTS superconducting filter, and the cryogenic low noise amplifier. The proposed eight-resonator HTS hairpin filter with $2 \%$ bandwidth at X-band frequency shows low insertion loss (below1 dB) and good skirt rejection (down to $60 \mathrm{~dB}$ at $50 \mathrm{MHz}$ from the pass-band edge). The two-stage low noise amplifier is designed by commercial low noise HEMT devices, NE321 000. Two Lange couplers are located at output and input ports of each amplifier stage to improve the return loss and to make LNA easily match to optimal low noise impendence. The cryogenic amplifier depicts a gain of $30 \mathrm{~dB}$ and an NF of 0.4 $\mathrm{dB}$ at $77 \mathrm{~K}$ in X-band frequencies.

A very high rejection is needed to eliminate the unwanted spurious signals. The acceptable specification is $110 \mathrm{~dB}$ rejection at $500 \mathrm{MHz}$ away from the band-edge of the filter. In general, the skirt rejection of a hairpin HTS filter is pretty good near the pass-band edge, but may become worse far from the pass-band edge. To meet the requirement of this commercial radar application, a high rejection suspended stripline filter with five resonators is put after the low noise amplifier to satisfy the demand. It works in room temperature and shows a quit large rejection of $80 \mathrm{~dB}$ at $500 \mathrm{MHz}$ away from the pass-band edge. The whole module shows an NF of $1.5 \mathrm{~dB}$, a gain of $25 \mathrm{~dB}$ within pass-band frequencies, and a rejection of better than $110 \mathrm{~dB}$ at $500 \mathrm{MHz}$ away from pass-band edge. It totally solves the interference problem in this dual mode radar.

There still have rooms for each individual component to be fine tune to their best performance. In fact, we just simply try to prove that the proposed front end can solve the interference problem effectively.

\section{High TEMPERATURE SUPERCONDUCTOR FILTER}

Basically, a coupled-resonator filter is composed by resonators serially coupled to each other and the first and last resonators are coupled to the input and output ports. If the BPF parameters and LPF prototype values are known, the external Q factor and coupling coefficient can be obtained as follows [1]:

$$
\mathrm{Q}_{\mathrm{a}}=\frac{\mathrm{g}_{0} \mathrm{~g}_{1}}{\mathrm{w}} \quad \mathrm{Q}_{\mathrm{b}}=\frac{\mathrm{g}_{\mathrm{n}} \mathrm{g}_{\mathrm{n}+1}}{\mathrm{w}}:
$$

where $\mathrm{Q}_{\mathrm{a}}$ is the external $\mathrm{Q}$ factor for input port, $\mathrm{Q}_{\mathrm{b}}$ is the external $Q$ factor for output port, $g_{0} \ldots \ldots g_{n+1}$ are the Chebyshev low-pass prototype values, $\mathrm{w}$ is the fractional bandwidth

$$
\mathrm{K}_{\mathbf{j}, \mathbf{j}+1}=\frac{w}{\sqrt{g_{j} \cdot g_{j \div 1}}}
$$

Where the $K_{j, j+1}$ is the coupling coefficient between $j$ 's and $\mathrm{j}+1$ 's resonator. 


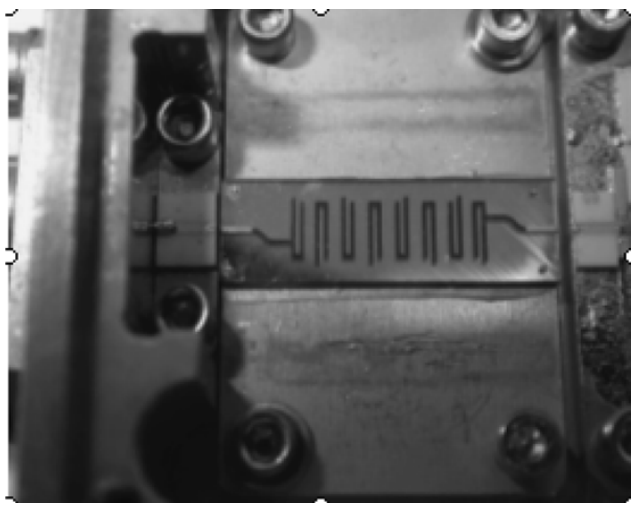

Fig. 1. The photo of HTS superconductor filter.

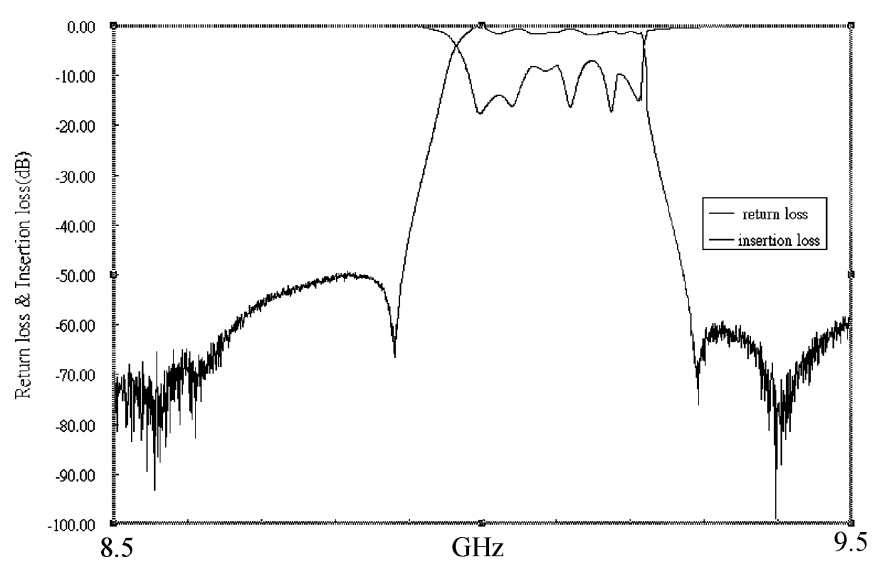

Fig. 2. Measured return loss and insertion loss of proposed HTS filter realized on 20 mil $\mathrm{LaAlO}_{3}$ substrate (including connectors and transitions).

Traditionally, there are two methods to design filter from the known external $\mathrm{Q}$ factors and coupling coefficients. The first method [2] is to derive the formula to calculated $\mathrm{Z}_{\mathrm{oo}}$ (odd mode impendence) and $\mathrm{Z}_{\mathrm{oe}}$ (even mode impendence). The width and gap of the coupled lines can be obtained from these impedances by commercial software such as Lincalc. The design procedures are complicated, time-consuming, and limited by the geometry of resonators. The second method [3] is to obtain external Q values and coupling coefficients by measurement. Then, appropriately places each resonator according to the experimental results. It is still time consuming and strongly depending on fabrication process.

In recent years, the commercial 2.5D or 3D EM simulator becomes to be the best choice to design filters. The external Q values and coupling coefficients can be obtained by EM simulation. Then, put them together and to get the whole filter response by EM simulation. Finally, gets the optimal performance by fine-tuning.

Due to its compact size and good rejection, the hairpin filter is chosen [4]. The photo of proposed eight-resonator HTS hairpin filter is shown Fig. 1 where the substrate is 20 mil $\mathrm{LaAlO}_{3}$. The tapped-line is used as input and output coupling structure. The measured performances without tuning are shown in Fig. 2. The passband insertion loss is approximately to be $0.2 \mathrm{~dB}$ at frequencies where the return loss is better than $15 \mathrm{~dB}$. The worst passband insertion loss is around $1 \mathrm{~dB}$ that happens at worst return loss points. The passband return loss could be improved

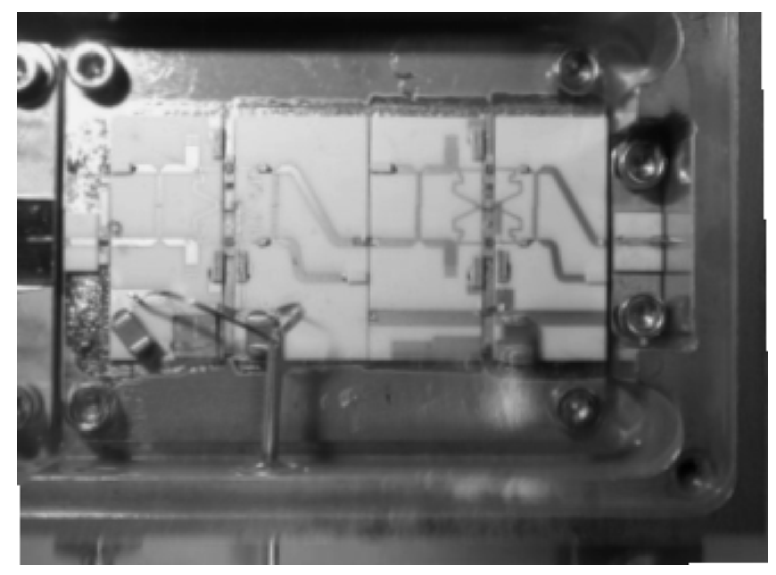

Fig. 3. Photo of cryogenic low noise amplifier.

LNA $1.5 \mathrm{~V} ; 24 \mathrm{~mA} ; 77 \mathrm{~K}$

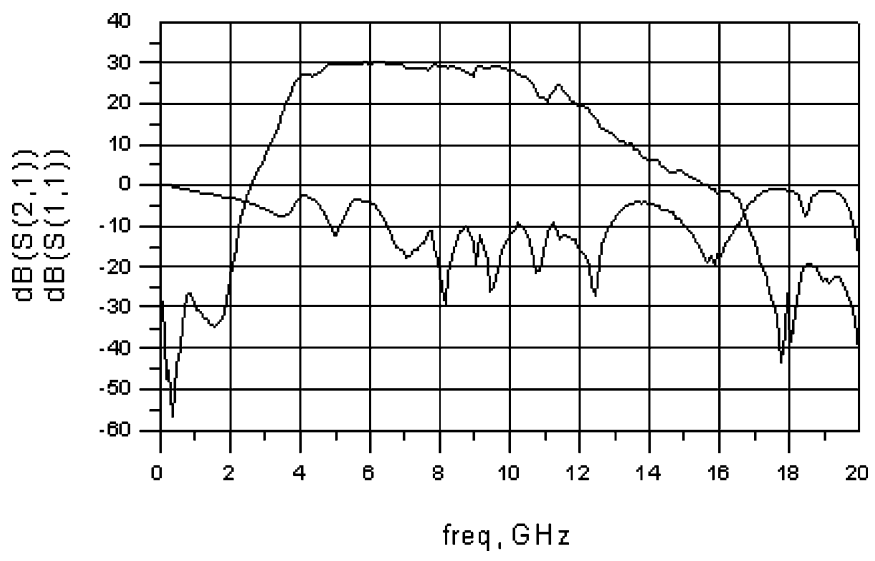

Fig. 4. Measured return loss and gain of cryogenic low noise amplifier at $77 \mathrm{~K}$.

by adding tuning mechanics. It can be observed in Fig. 2 that some transmission zeros exist in lower and higher rejection band which make the rejection skirt steep. They come from the cross coupling of nonadjacent resonators [5], [6].

\section{THE CRYogenic Low NoISE AMPLIFIER}

The NF of HEMTs reduces and the gain of HEMTs increases as the temperature becomes lower. Both are beneficial to low noise amplifier, except higher gain is possible to induce unwanted oscillation. Because the modern HEMT devices have very good gain, the most important thing in cryogenic LNA design is to avoid the unwanted oscillation. The photo of LNA is shown in Fig. 3. The LNA are self-biased with single power supply. The chocks in LNA comprise a quarter wavelength high impendence transmission line, a bypassing capacitor, and a stability resistor. Two Lange couplers are located at input and output of each LNA stage. The Lange coupler keeps the return loss of LNA good, so that each individual HEMT could be matched close to its optimal NF impedance.

The gain and return loss of the LNA at $77 \mathrm{~K}$ are shown in Fig. 4. From $8.5 \mathrm{GHz}$ to $9.5 \mathrm{GHz}$, the gain is around $30 \mathrm{~dB}$. Return loss is better than $10 \mathrm{~dB}$. The increasing of gain corresponding to room temperature is about $4 \mathrm{~dB}$. The return loss shows very little change. Some little dips exist in gain curve. 


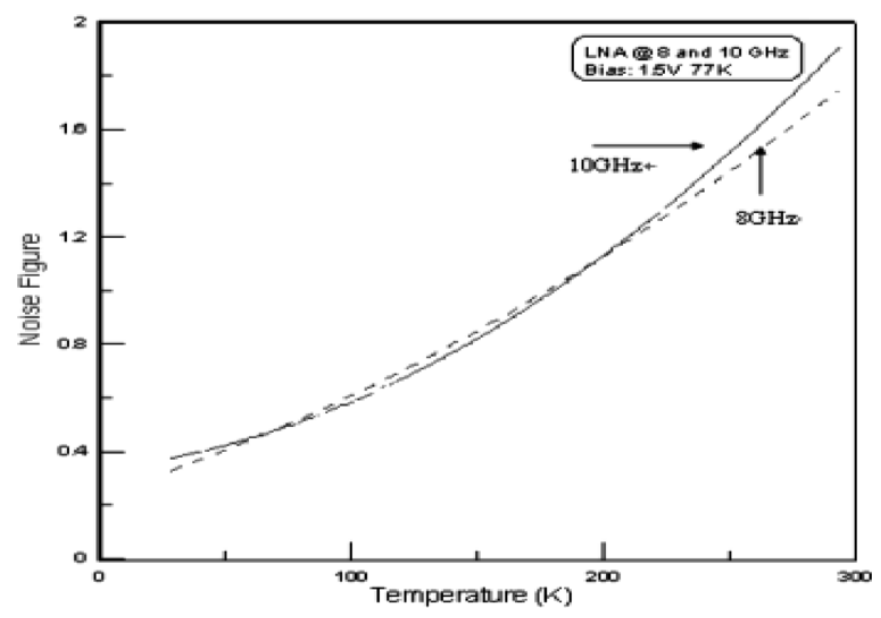

Fig. 5. Variation of gain and noise figure of cryogenic LNA vs. temperature.

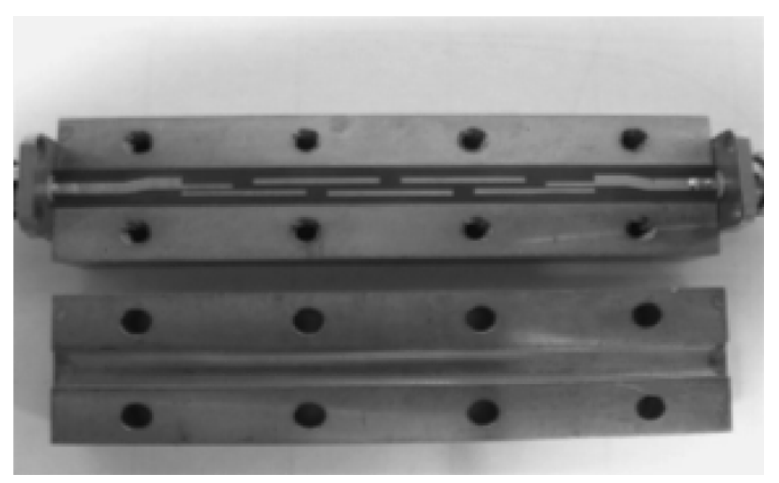

Fig. 6. Photo of parallel coupled filter fabricated by suspended line.

The dips possibly come from the discontinuity caused by circuit assembly. The variation of gain and NF versus temperature is shown in Fig. 5. As temperature changes from room temperature to $77 \mathrm{~K}$, the $\mathrm{NF}$ reduces from $1.8 \mathrm{~dB}$ to $0.4 \mathrm{~dB}$. This is a big improvement for whole system.

\section{The SySTEM MEASUREMENT}

In order to eliminate the interference completely, the rejection must be greater than $110 \mathrm{~dB}$ at $500 \mathrm{MHz}$ away from the passband edge of the superconductor filter. As shown in Fig. 2, although the HTS filter shows rejection over $85 \mathrm{~dB}$ near the passband edge, it degrades to about $70 \mathrm{~dB}$ at frequency $500 \mathrm{MHz}$ away from passband edge. A suspended stripline filter having good rejection at $500 \mathrm{MHz}$ from pass-band edge is used after the amplifier. This parallel coupled filter with 5 resonators works in the same frequency range as the superconductor filter. The photo of the suspended stripline filter is shown in Fig. 6. Shown in Fig. 7(a) are measured results of the filter. The passband insertion loss is around $2.5 \mathrm{~dB}$. The return loss is better than $15 \mathrm{~dB}$. The rejection is over $80 \mathrm{~dB}$ at $500 \mathrm{MHz}$ from band-edge. Furthermore, there are no spurious and harmonics existing from 40 $\mathrm{MHz}$ to $20 \mathrm{GHz}$ as shown in Fig. 7(b).

The whole module includes front end and the suspended filter is shown in Fig. 8. A $3 \mathrm{~dB}$ attenuator is put after the LNA to increase the stability of the whole module. The front end works within a cryogenic refrigerator, but the suspended filter works

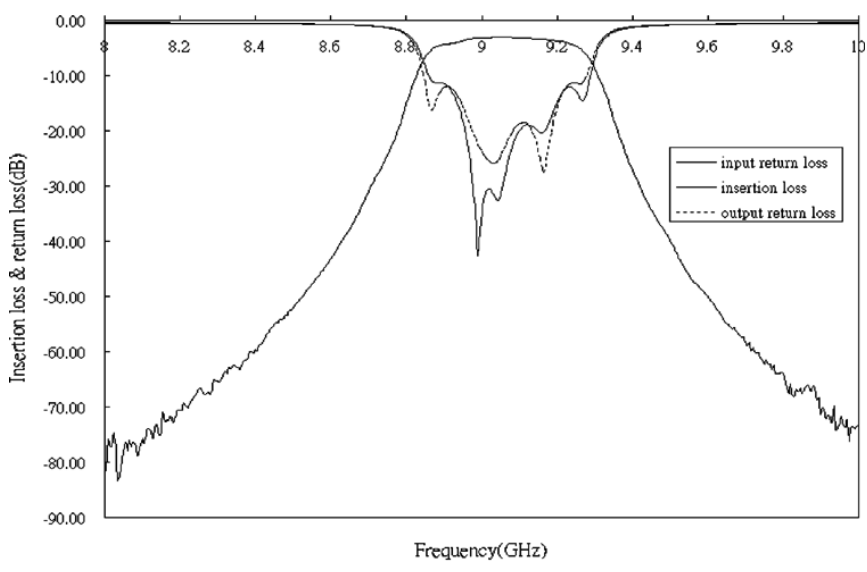

(a)

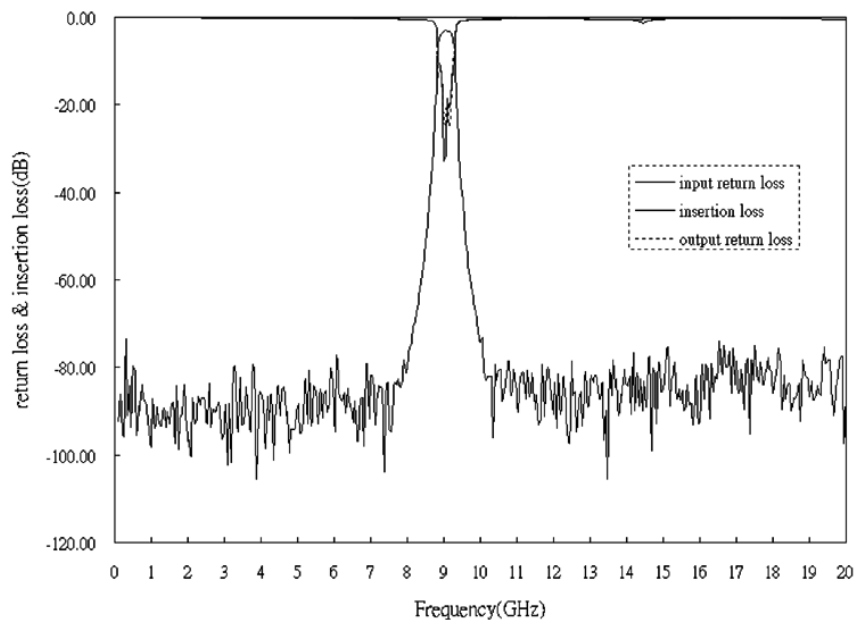

(b)

Fig. 7. Measured insertion loss and return loss of suspended line filter. (a) Pass-band ( $8 \mathrm{GHz}-10 \mathrm{GHz})$ (b) rejection band (40 MHz-20 GHz).
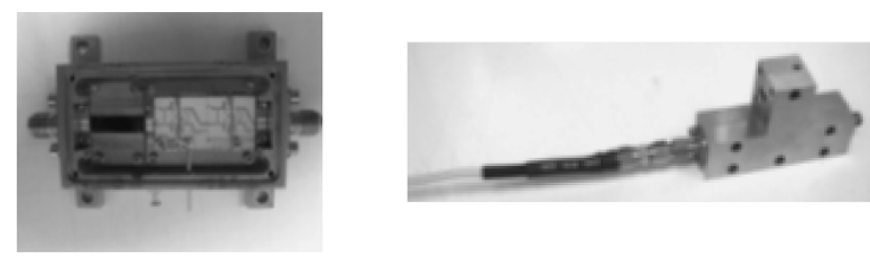

Fig. 8. Photo of the whole module.

outside the refrigerator in room temperature through a $30 \mathrm{~cm}$ long coaxial cable.

The overall measured NF of the whole module is around $1.5 \mathrm{~dB}$ in the passband. Fig. 9(a) shows the gain of the whole modules which is typically $25 \mathrm{~dB}$ in the passband. The return loss is better than $10 \mathrm{~dB}$ at most part of the pass-band of filter. It is estimated that the total rejection should be larger than $140 \mathrm{~dB}$. Because of the dynamic range limitation of network analyzer, the measured value is only around $80 \mathrm{~dB}$. Again, Fig. 9(b) shows that there exists no spurious and harmonics from $40 \mathrm{MHz}$ to $20 \mathrm{GHz}$.

Finally, as shown in Fig. 10, the whole module is inserted into the receiver port of the searching radar. All the transmitters are turned on and the transmitter signals are pulse modulated. The spectrum analyzer is put at position A and B in Fig. 10 to 


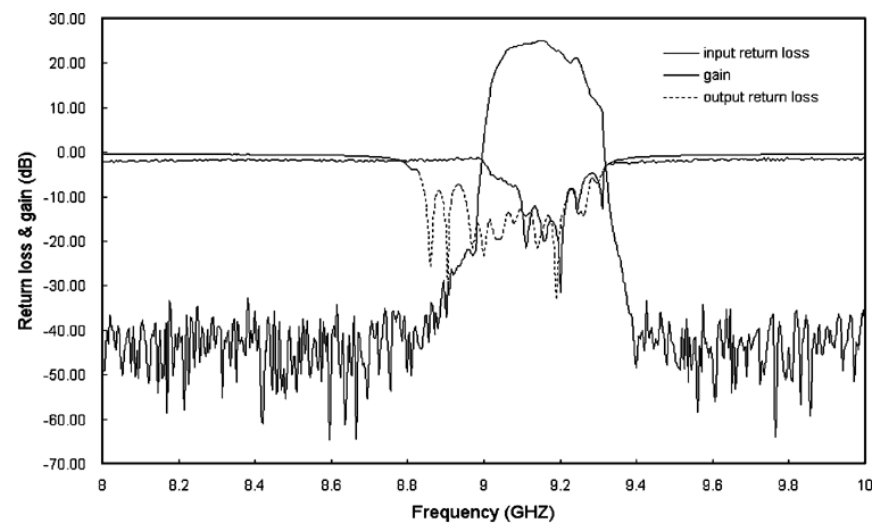

(a)

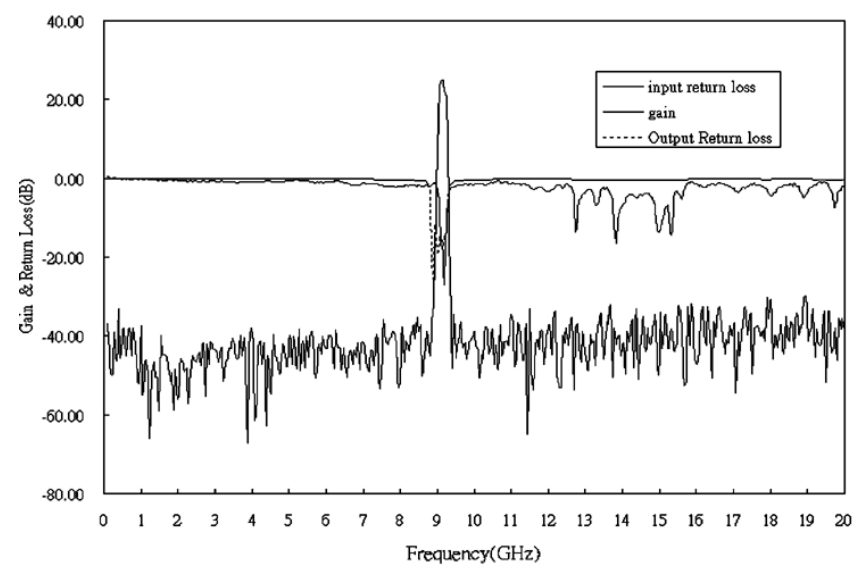

(b)

Fig. 9. Measured results of whole modules. (a) Narrow band. (b) Broadband.

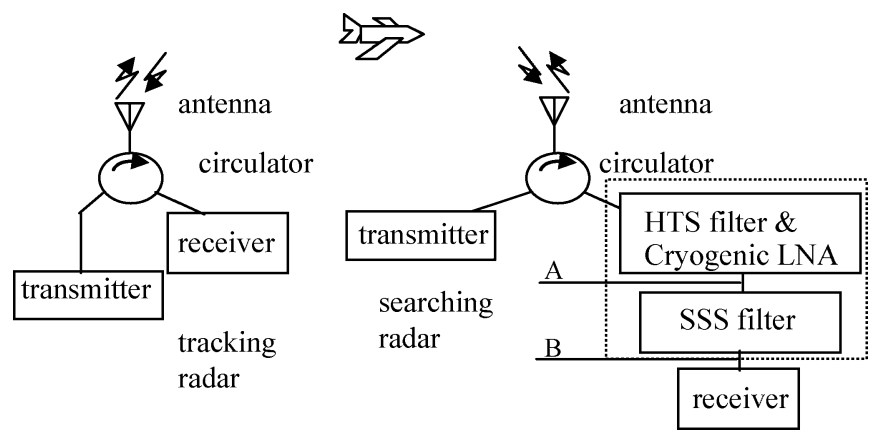

Fig. 10. Configuration of measurement for interference of dual mode radar.

measure the interference signal. It is clear that most of the signals coming from tracking radar are eliminated, and only the signals coming from the transmitter of the searching radar are received as shown in Fig. 11(a) and (b). The received spectrum at position B from $8.2 \mathrm{GHz}$ to $9.2 \mathrm{GHz}$ is shown in Fig. 11(a). The spectral shape of the signal is the standard pulse modulated signal sending from the transmitter of searching radar. The interferences caused by tracking radar do not appear. The Fig. 11(b) shows a broad-band view of the measured result at position A. There exists the unwanted second harmonic signal in this figure. The reason is due to the nonlinearity of the LNA. The nonlinearity causes the second harmonic signal to appear.

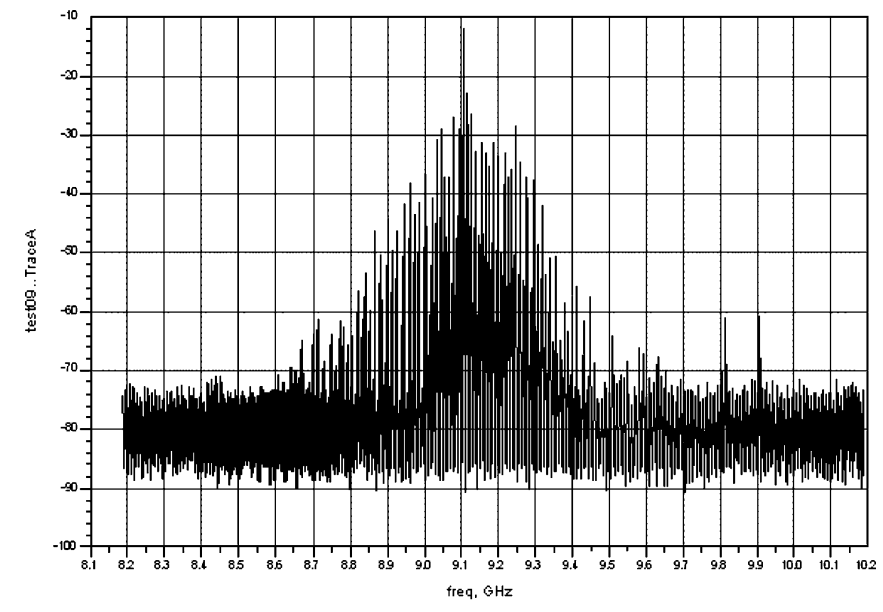

(a)

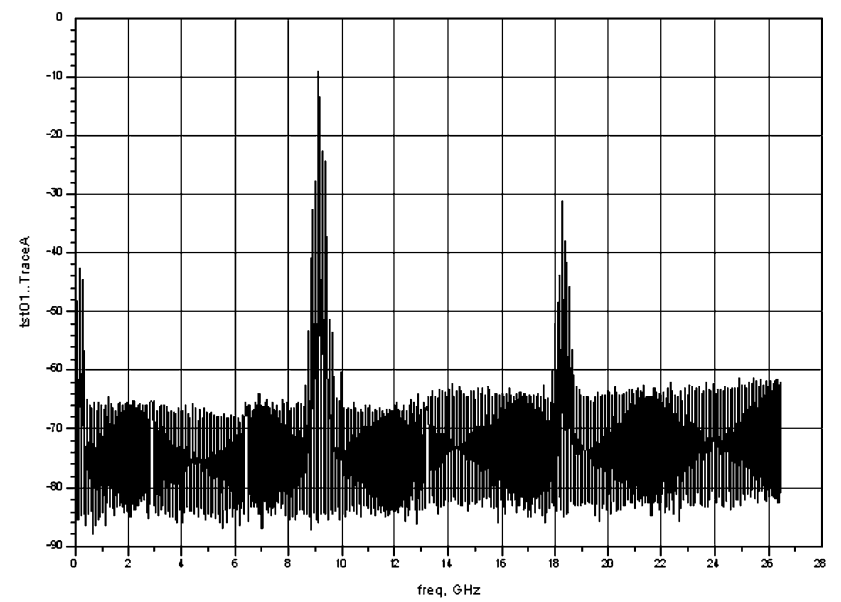

(b)

Fig. 11. System measurer result after the whole modules is inserted. (a) Narrow band. (b) Broadband.

\section{CONCLUSION}

A module includes HTS superconducting filter, cryogenic low noise amplifier and an external suspended line filter has been designed to eliminate the interference between searching and tracking radar. The module has been inserted into the receiving path of searching radar. Although there still have rooms for each individual component to be fine-tuned to its best performance, pretty good interference-eliminating capability has been observed.

\section{REFERENCES}

[1] G. L. Matthai, L. Young, and E. M. T. Jones, Microwave Filter, Impendence-Matching Networks, and Coupling Structure. Norwood, MA: Artech House, 1975, ch. 8, pp. 434-472.

[2] S. B. Cohn, "Parallel-coupled transmission-line filter," IRE Trans. Microw. Theory Tech., vol. MTT-6, pp. 223-231, Apr. 1958.

[3] J. S. Wong, "Microstrip tapped-line filter design," IEEE Trans. Microw. Theory Tech., vol. MTT-27, no. 1, Jan. 1979.

[4] E. G. Cristal and S. Frankel, "Design of hairpin-line and hybrid hairpin parallel-coupled-line filter," in IEEE Microw. Theory Tech. Symp. Dig., 1971, pp. 12-13.

[5] R. Levy, "Filter with single transmission zeros at real and imaginary frequencies," IEEE Trans. Microw. Theory Tech., vol. MTT-24, pp. 172-181, 1976.

[6] J.-S. G. Hong and M. J. Lancaster, Micro-Strip Filters for RF/Microwave Application. New York: Wiley, 2001, ch. 9, pp. 299-306. 